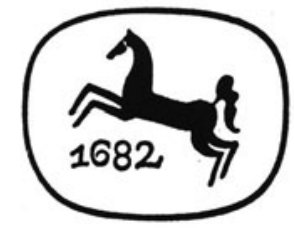

Linguistik. Ein Handbuch 


\title{
Linguistik
}

\section{Ein Handbuch}

Herausgegeben von

\author{
AndRÉ Martinet \\ unter Mitarbeit von Jeanne Martinet \\ und Henriette Walter
}

MCMLXXIII

J. B. Metzlersche Verlagsbuchhandlung

Stuttgart 
Aus dem Französischen übersetzt von Irmela Rehbein (Kap. 1-25) und Steffen Stelzer (Kap. $26 \mathrm{ff}$.), mit einer bibliographischen Ergänzung versehen von Fritz Tangermann.

ISBN 978-3-476-00221-1

ISBN 978-3-476-02989-8 (eBook)

DOI 10.1007/978-3-476-02989-8

(c) 1969, by Éditions Denoël, Paris 7c

(c) der deutschen Übersetzung 1973, by Springer-Verlag GmbH Deutschland Ursprünglich erschienen bei J. B. Metzlersche Verlagsbuchhandlung und Carl Ernst Poeschel Verlag GmbH in Stuttgart 1973 


\section{Vorbemerkung}

Diese Kollektivarbeit ist aus Seminararbeiten hervorgegangen, die während des Studienjahres 1966-67 unter der Leitung von André Martinet in dessen Eigenschaft als Direktor der Etudes de linguistique structurale an der Ecole Pratique des Hautes Etudes (Paris) fertiggestellt worden sind. Die Aufteilung der allgemeinen Linguistik, so wie sie heute verstanden wird, in einige Dutzend verschiedene Arbeitsgebiete ist in Ubereinstimmung mit den engsten Mitarbeitern vorgenommen worden. Die sorgfältige Bearbeitung der Erstfassung eines jeden Kapitels wurde jeweils einem der Seminarteilnehmer überantwortet, und darüberhinaus in zehn von 51 Fällen ehemaligen Schülern oder Kollegen anvertraut. Lektüre und Diskussion eines jeden Kapitels sollten dabei grundsätzlich während der Seminarsitzungen erfolgen. Dies konnte jedoch nur für etwa die Hälfte der Kapitel geschehen. Die rechtlichen, zu spät abgeschlossenen Kapitel wurden von Arbeitsgruppen geprüft, die sich ausschließlich aus Mitarbeitern an diesem Band zusammensetzten und die sich während des Juni 1967 mehrfach wöchentlich trafen. Dabei verdienen Rita Chademony, Blanche-Noëlle Grunig, Gabriel Bès, Alfredo Hurtado und Georges Kassai als die eifrigsten Mitarbeiter besondere Erwähnung. Es gibt kein Kapitel in diesem Band, das nicht von der Anstrengung zeugt, die einzelnen Teile besser zu koordinieren und das Werk einheitlich zu gestalten. Allerdings mußte sich der Herausgeber, ohne der Praxis des re-zeriting der amerikanischen editorischen Gepflogenheiten zu entsprechen, bei einer Gesamtrevision des Manuskripts an der einen oder anderen Stelle einen Eingriff vorbehalten, insbesondere da, wo der ursprünglich eingeschlagene Weg der Darstellung verlassen worden war. Jeanne Martinet, Henriette Walter und Gérard Walter haben sich der mühevollen und schwierigen Aufgabe unterzogen, die Indices zu erstellen und die weiterführende Bibliographie auf den letzten Stand der linguistischen Forschung zu bringen.

Nach Möglichkeit wurde in dem vorliegenden Band versucht, von allen Theorien und Tendenzen der zeitgenössischen Linguistik einen getreuen Eindruck zu geben, der dem Rahmen des Bandes entsprechend vollständig sein sollte. Dennoch kann kein Zweifel daran bestehen, daß der Band von Ort und Zeitpunkt seines Entstehens geprägt ist. Es handelt sich um das Produkt eines Seminars, dessen Teilnehmer überwiegend aus Mitteleuropa stammten und die gerade in die Prinzipien der strukturalen Linguistik eingeführt worden waren; zum großen Teil waren sie daran gewöhnt, die linguistische Problematik unter dem Blickwinkel der funktionalen Linguistik zu betrachten. Unter diesen Umständen war es schwierig, zumindest unterschwellig einige Vorentscheidungen auszuschließen, wie zum Beispiel in der Bibliographie, wo die Vielzahl der zitierten wissenschaftlichen Untersuchungen des Herausgebers eine große Vertrautheit seiner Schüler beweist. Dennoch hoffen Herausgeber wie Mitarbeiter, daß Leser wie Benützer des Bandes denselben Eindruck der Neutralität gewinnen, dessen sie sich bei der Abfassung des Kapitels befleißigt haben.

$\mathrm{Zu}$ einem Zeitpunkt, in dem zumindest in einzelnen linguistischen Teilgebie- 
ten eine rege Forschertätigkeit zu verzeichnen ist, sind gewisse Veränderungen unerläßlich und daher hat der deutsche Verlag in Übereinstimmung mit dem Herausgeber und den Autoren Fritz Tangermann dazu veranlaßt, die weiterführende Bibliographie an allen denjenigen Stellen zu ergänzen, wo es geboten schien. Ihm und den Übersetzern Irmela Rehbein und Steffen Stelzer sei an dieser Stelle. ausdrücklich gedankt. Von seiten des deutschen Verlages hat man es bedauert, daß das Handbuch kein Kapitel „Soziolinguistik“ enthält, weil sich die Soziolinguistik und die damit verbundenen Fragestellungen und Aufgaben in der Bundesrepublik zu einem Schwerpunkt der linguistischen Forschung entwickelt haben. Indessen werden sich in Kapitel 39 (Plurilinguismus und Interferenzen) und in Kapitel 51 (Verschiedenheit der Idiome) genügend Stichpunkte finden, die annähernd den soziolinguistischen Bereich decken.

André Martinet 


\section{Inhaltsverzeichnis}

Vorbemerkung .................

Spracherwerb (Andrée Tabouret-Keller) . . . . . . . . . . . . 1

Syntaktische Autonomie und Klassifizierung der Moneme (Denise François) 5

Sprechkontinuum: Die Syntagmatik (Oswald Ducrot) . . . . . . . . . . 10

Wortwahl des Sprechers: Die Paradigmatik (Jean-René Reimen) . . . . . 18

Vergleich und Rekonstruktion (Jacqueline Manessy-Guitton) . . . . . . 27

Zusammensetzung und Ableitung (Henriette Walter) . . . . . . . 35

Kontakte, Konvergenzen, Divergenzen (Fanny de Sivers) . . . . . . . . 39

Kontext und Situation (Frédéric François) . . . . . . . . . . . . 42

Diachronie (Giovanna Madonia) . . . . . . . . . . . . . . . 49

Okonomie (Giovanna Madonia) . . . . . . . . . . . . . . . . . . . 55

Aussage (Joseph Verguin) . . . . . . . . . . . . . . . . . . . . . 60

Ethno-Linguistik (Jacqueline Schön) _ . . . . . . . . . . 63

Erweiterung, Koordination, Subordination (Georges Kassai) . . . . . . 68.

Funktionen der Sprache (Denise François) . . . . . . . . . . . . . 71

Grammatikalische Funktionen (Mortéza Mahmoudian) . . . . . . . . 77

Form und Substanz (Gabriel Bès) _. . . . . . . . . . . . . . . 8

Grammatik (Claude Hagège) . . . . . . . . . . . . . . . . . 88

Generative Grammatiken und Transformation (Blanche-Noëlle Grunig) . . 95

Schrift (Maurice Coyaud) . . . . . . . . . . . . . . . . . . 105

Information (Frédéric François) . . . . . . . . . . . 111

Sprache (Georges Mounin) . . . . . . . . . . . . . . . . . . 117

Sprache und Rede (Gabriel Bès) _. . . . . . . . . . . . . . . . . 125

Künstliche Sprachen (Maurice Coyaud) _. . . . . . . . . . . . . 131

Lexikon (Paul Pupier) . . . . . . . . . . . . . . . . 136

Wissenschaftsgeschichte der Linguistik (Georges Mounin) . . . . . . . . 147

Angewandte Linguistik (André Martinet) . . . . . . . . . . . 152

Geographische Linguistik und Dialektologie (Jean-Francis Lacroix) . . . 157

Quantitative Linguistik (Blanche-Noëlle Grunig) . . . . . . . . . . . 164

Logik und Sprache (Oswald Ducrot) . . . . . . . . . . . . . . . . . 171

Merkmal (Soc-Kiou Tschen) _. . . . . . . . . . . . . . . 176

Morphologie (Tsutomu Akamatsu) . . . . . . . . . . . . . 180

Wort (Fanny de Sivers) . . . . . . . . . . . . . . . . . 185

Neutralisierung (Hubert Maes) _. . . . . . . . . . . . 189

Pathologie der Sprache (David Cohen) . . . . . . . . . . . . . 195 
Phonematik (Claude Hagège) . . . . . . . . . . . . . . 200

Phonem (Gabriel Bès) . . . . . . . . . . . . . . . . . . . . . . . 206

Phonetik (Rita Chademony) . . . . . . . . . . . . . 215

Phonologie (Claude Hagège) . . . . . . . . . . . . . . . . . . 221

Plurilinguismus und Interferenzen (Andrée Tabouret-Keller) . . . . . 226

Prosodie (Claude Hagège) ． . . . . . . . . . . . . . . . . . . . . 231

Psycholinguistik (Paul Wald) . . . . . . . . . . . . 236

Redundanz (Frédéric François) . . . . . . . . . . . . . . . . 246

Sinn (Georges Kassai) . . . . . . . . . . . . . . 251

Zeichen (Mortéza Mahmoudian) . . . . . . . . . . . . 258

Stil und Stilistik (Aline Levavasseur) . . . . . . . . . . . 265

Synchronie (Alfredo Hurtado) . . . . . . . . . . . . . . . . . . . 272

Syntax (Alfredo Hurtado) . . . . . . . . . . . . . . . . . . 275

Ubersetzung (Georges Mounin) . . . . . . . . . . . . . . . . . . 282

Typologie (Elisabeth Berten) ． . . . . . . . . . . . . . . . . . 286

Varianten $(T$ sutomu Akamatsu, . . . . . . . . . . . . . . . . . . . . 290

Verschiedenheit der Idiome (Marthe Philipp) . . . . . . . . 295

Weiterführende Bibliographie . . . . . . . . . . . 300

Literaturverzeichnis . . . . . . . . . . . . . . . . . . 338

Register . . . . . . . . . . . . . . . . 368 